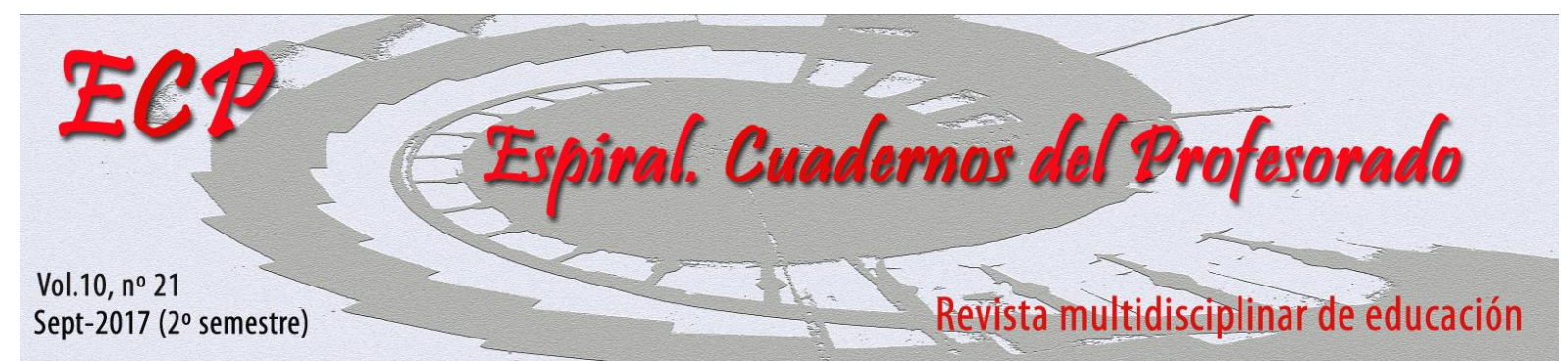

\title{
La presencia del concepto de RSC en los sitios web de las empresas de turismo activo de Andalucía y su valor para los centros educativos escolares
}

\section{The CSR concept presence in Andalusian active tourism websites and its value to schools centers}

\author{
Marta Pérez-Villalba $^{1}$, Jesús Fernández-Gavira ${ }^{2}$, y Pablo Caballero Blanco ${ }^{2}$ \\ 1. Grupo de investigación Gestión e Innovación en Servicios Deportivos, Ocio, Recreación y Acción \\ Social (GISORAS), Universidad de Sevilla, España \\ 2. Departamento de Educación Física y Deporte, Universidad de Sevilla, España
}

\begin{abstract}
RESUMEN: La Responsabilidad Social Corporativa (RSC) es una manera de gestionar que va más allá de las obligaciones legales de las organizaciones, se trata de garantizar un desarrollo sostenible en tres áreas principales: económica, social y medio ambiental. Este artículo analiza la presencia del concepto de RSC en el contenido de los sitios web de las empresas de turismo activo de Andalucía. Teniendo en cuenta que para este tipo de empresas la población escolar es un importante nicho de mercado, cobra especial relevancia saber si éstas comunican sus acciones de RSC. El método de estudio se ha basado en un análisis cuantitativo del contenido semántico de 310 sitios webs de empresas de turismo activo a través de la búsqueda de la palabra clave "Responsabilidad Social Corporativa" y sus sinónimos mediante el motor de búsqueda Google. Los resultados indican una escasa presencia del concepto de la RSC en el contenido web, así como poca visibilidad de acuerdo a su ubicación. La adopción de una gestión desde la RSC contribuiría a la educación integral de niños y jóvenes, y su comunicación efectiva, aportaría un valor añadido para los docentes en la selección de proveedores de actividad física en el medio natural.
\end{abstract}

Palabras clave: Responsabilidad Social Corporativa, desarrollo sostenible, turismo activo, deporte, Educación Física.

\begin{abstract}
Corporate Social Responsibility (CSR) is a management practice that goes beyond organizations legal obligations, it aims to guarantee a sustainable development in three main areas: economic, social and environmental. This article analyses the presence of CSR concept in the content of Andalusian active tourism companies' websites. Taking into account that for this type of business the school population is an important market niche, particular importance is attached to know if companies communicate their CSR actions. The methodology is based on a quantitative analysis of the semantic content of 310 Andalusian active tourism companies' websites through the keyword search "Corporate Social Responsibility" and its synonyms by using Google search engine. The results indicate a scarce presence of the CSR concept within websites content, as well as a low visibility of it according to its location. Adopting a CSR management approach would contribute to a comprehensive education of children and youth, and its effective communication would add value to teachers' decision when selecting an outdoor physical activity provider.
\end{abstract}

Key words: Corporate Social Responsibility, sustainable development, active tourism, sport, Physical Education.

Pérez-Villalba, M., Fernández-Gavira, J., y Caballero Blanco, P. (2017). La presencia del concepto de RSC en los sitios web de las empresas de turismo activo de Andalucía y su valor para los centros educativos escolares. Espiral. Cuadernos del Profesorado, 10(21), 118-125. 


\section{Introducción}

En los últimos años, el concepto de la Responsabilidad Social Corporativa (RSC) ha cobrado fuerza en el ámbito del turismo debido a las fuertes presiones por parte de los stakeholders (trabajadores, clientes, proveedores, medios de comunicación, etc.) para que se adopten prácticas sostenibles (Fatma, Rahman, \& Khan, 2016) que eviten, minimicen o contrarresten los posibles impactos negativos del turismo en los territorios, tanto desde un punto de vista social, medioambiental y económico (Elkington, 2004). De esta manera, se asegurará un desarrollo sostenible del sector que satisfaga "las necesidades de la generación presente sin comprometer la capacidad de las generaciones futuras para satisfacer sus propias necesidades" tal y como recogió la Comisión Mundial sobre el Medio Ambiente y el Desarrollo de Naciones Unidas en su informe Nuestro Futuro Común (World Commission on Environment and Development, 1987). Diversas son las definiciones atribuidas al concepto de la RSC (también llamada Responsabilidad Social Empresarial o Desarrollo Sostenible), tanto por autores académicos (Bowen, 1953; Carroll, 1979) como por organismos oficiales (Comisión Europea, 2001), agencias multilaterales (United Nations, 2014) u organizaciones sin ánimo de lucro (Forética, 2002; Observatorio de Responsabiliad Social Corporativa, 2014). Una de las definiciones consideradas como pioneras es la de Bowen que se refiere a "las obligaciones del empresario de perseguir las políticas, tomar las decisiones y seguir líneas de acción que sean deseables para los objetivos y los valores de la sociedad" $(1953$, p. 6). Por su parte, Carroll $(1979,1991)$ propone una definición basada en cuatro categorías de responsabilidades que han de asumir las empresas: económicas (la responsabilidad básica de obtener beneficios y, a la vez, ser viable), legales (el deber de obedecer la ley), éticas (responsabilidad de actuar de manera coherente con la sociedad) y discrecionales (actividades que van más allá de las expectativas de la sociedad). A pesar de las distintas definiciones, la mayoría abordan la RSC desde un enfoque integral que no sólo cubre la parte medioambiental, hasta ahora la más tratada en empresas de turismo activo, sino que también incluye la parte social y económica, a la vez que abogan por mantener un discurso bidireccional con los stakeholders.

Desde el ámbito de la actividad física en el medio natural (AFMN), algunos autores se han hecho eco de la urgencia de concienciar a las empresas sobre la necesidad de incluir la RSC en sus prácticas. Quintana y Gregory (2013) señalan la existencia de malas prácticas en el desarrollo de actividades deportivas y recreativas en el medio natural, especialmente en zonas débiles o de especial protección, que llevan a un consumo incontrolado de recursos naturales y a la extralimitación de la capacidad de carga en épocas determinadas, lo que resulta en una degradación de los espacios. Esta inquietud sobre la fragilidad de los espacios naturales destinados al turismo activo también la puso de manifiesto la Organización Mundial del Turismo en su el Informe Global sobre Turismo de Aventura (Perdomo, 2014). Por su parte, Funollet, Inglés, \& Labrador (2016), reclaman la promoción de programas para la educación de comportamientos y actitudes a través de los centros educativos y de los medios de comunicación para concienciar a las futuras generaciones de practicantes y proveedores de turismo activo. En el año 2013 se llevó a cabo un estudio sobre la calidad de las empresas españolas de turismo activo. Con este fin, se utilizó la herramienta valorativa HEVA que en su tercer bloque de contenidos aborda la identificación del nivel de importancia de siete parámetros que muestran la calidad técnica en el servicio de turismo activo, entre los que se incluye el medio ambiente. De los parámetros evaluados, el que recibió una menor puntuación fue el de medio ambiente (Mediavilla \& Gómez, 2016). Teniendo en cuenta la tendencia hacia un consumidor más sensibilizado con el entorno el cual incorpora consideraciones sociales, medioambientales y éticas en su decisión de compra (Galan, Galera, \& Valero, 2017), la implementación de la RSC en la gestión de las empresas de turismo activo puede servir como cauce para mejorar la competitividad y la reputación de las empresas, respondiendo así a la demanda de productos y servicios socialmente responsables (Kastenholz, 2009). De esta manera, conseguir una reputación de empresa socialmente responsable, aportará una ventaja competitiva y un valor añadido, especialmente en la organización de actividades dirigidas a los escolares, pues estas requieren de equidad, transparencia, participación, desarrollo e identificación, Plan Xertatu (2007). Además, la AFMN se presentan como un contexto privilegiado para promover el desarrollo positivo de los niños y jóvenes (capacidades como la responsabilidad, 
empatía, autonomía, etc.), y especialmente para promover la educación ambiental (Caballero y Delgado, 2014; Baena y Granero, 2014; Baena, Granero y Ortiz, 2012). Por lo tanto, es necesario que las empresas de turismo activo que ofertan actividades para niños y jóvenes (en contexto de educación formal y no formal), definan una serie de acciones, metodología y objetivos, que contribuyan a la educación social y ambiental de los participantes desde las AFMN (Baena, Gómez y Granero, 2008). A su vez, las empresas deben mostrar coherencia y no sólo educar bajo los principios de la responsabilidad social, si no también demostrar que los principios forman parte de su manera de gestionar y actuar.

La comunicación de la RSC es utilizada tanto a nivel interno como externo para informar a todos los stakeholders de una organización (trabajadores, clientes, proveedores, medios de comunicación, etc.) de las acciones llevadas a cabo en materia de RSC y así ganar su compromiso (Cornelissen, 2014). Existen diversos instrumentos utilizados por las empresas para comunicar la RSC, incluyendo informes anuales, panfletos, publicidad o sitios webs corporativos. Los sitios webs se presentan como uno de los medios más utilizados como medio para informar sobre la RSC, pues poseen características particulares como la recuperación de documentos electrónicos, herramientas de búsqueda o la posibilidad de incorporar material multimedia, que permiten a las empresas comunicar de manera más específica (Saat \& Selamat, 2014). En el sector turístico, los sitios webs son considerados como la principal fuente de información para los clientes (Cao \& Yang, 2016; Corigliano \& Baggio, 2006) y por ello, requieren de sistemas para evaluar su eficacia en la presentación de la información que las personas usuarias desean y necesitan conocer. Actualmente, no existe un método universalmente aceptado o una técnica para la evaluación de sitios webs, pero sí multitud de propuestas que pueden ser clasificadas en dos sistemas: métodos de usabilidad heurísticos y métodos automatizados (Corigliano \& Baggio, 2006). Los métodos automatizados, consisten en utilizar un programa para capturar las características de la información presentada en un sitio web a través de un conjunto de criterios como la búsqueda de palabras clave (Cao \& Yang, 2016). En el marco de la RSC, se han llevado a cabo estudios mediante métodos automatizados para conocer la localización de los informes de RSC en los sitios webs corporativos (Hetze \& Winistörfer, 2016) o la presencia de palabras clave en el contenido de las webs (Sones, Grantham, \& Vieira, 2009).

Tal y como se desprende la revisión bibliográfica, la implementación y comunicación de la RSC es un área que cobra cada vez más importancia tanto en el sector del turismo y por ende, en el del turismo activo, y los sitios webs corporativos se presentan como uno de los instrumentos más válidos para su comunicación (Cornelissen, 2014). El sector del turismo activo, por la localización de su actividad en el medio natural, si no implementa una gestión a través de la RSC, está especialmente expuesto a crear un impacto negativo tanto en el territorio como en las comunidades que residen en éste. Además, el hecho de ofrecer actividades dirigidas a la población escolar crea la responsabilidad de educar en valores y de una manera ética (Baena, Gómez y Granero, 2008).

En línea con lo expuesto, el principal objetivo de este artículo es estudiar la presencia del concepto de la RSC en el contenido de los sitios webs de las empresas de turismo activo de Andalucía, así como detectar su ubicación dentro de los mismos. Con ello, se pretende aportar conocimiento sobre la existencia de una comunicación efectiva en materia de RSC que permita aportar un valor añadido tanto a las empresas de turismo activo, como a la industria en su totalidad, y a las actividades que se ofrecen a la población escolar.

\section{Material y método}

El enfoque metodológico adoptado para este estudio ha sido el análisis cuantitativo del contenido semántico de los sitios webs de las empresas de turismo activo de Andalucía a través de las palabras clave asociadas al concepto de la RSC: "responsabilidad social corporativa", "responsabilidad social empresarial" y "desarrollo sostenible". Diferentes autores han utilizado la búsqueda de palabras clave para analizar contenidos online (Basch, Zybert, Reeves, \& Basch, 2017; Evans, Chao, Leone, Finney, \& Fraser, 2016; Kwok, Singla, Phang, \& Lau, 2017; Sones et al., 2009). El proceso se ha llevado a cabo a través del motor de búsqueda Google que dispone de una función de búsqueda 
personalizada mediante operaciones booleanas que permiten rastrear aquellas páginas webs que incorporen las palabras deseadas (Ruiz \& Barnett, 2015). Mediante la incorporación de la palabra "site" antes de la dirección del sitio web se restringe la búsqueda de una palabra en un determinado sitio web. De esta manera, la petición "site: (dirección sitio web empresa turismo activo) RSC" lista las páginas del sitio web que contengan la palabra RSC. El navegador seleccionado ha sido Google Chrome, que mediante la utilización de la función modo incognito, ha permitido que Google no registrara el contenido visitado o descargado para así evitar que las búsquedas pudieran verse condicionadas por el histórico.

El universo de estudio lo conforman las 536 empresas de turismo activo registradas en la Federación de Empresas de Turismo Activo de Andalucía que disponen de un sitio web corporativo en activo. Se ha determinado que se necesita de una muestra de 310 empresas a evaluar para error de muestra del $+/-3.6 \%$ y un nivel de confianza del $95 \%$. Se ha aplicado el muestreo probabilístico mediante una selección sistemática de elementos muestrales que implica elegir dentro del universo $N$ un número $n$ de elementos a partir de un intervalo $K$ (Hernández, Fernández, \& Baptista, 2010). El intervalo se ha determinado en base al universo y la muestra a recoger dando como resultado la fórmula $K=N \ln$ que devuelve un intervalo de 2 .

$$
K=\frac{N}{n}=\frac{536}{310}=1.7(\text { redondeando }=2)
$$

La metodología aplicada en esta investigación plantea las siguientes limitaciones. En primer lugar, el motor de búsqueda Google sólo rastrea aquellas páginas que cumplen con sus criterios de búsqueda como por ejemplo la inclusión de un mapa web o de metatags. Por lo tanto, todas aquellas páginas que incumplan los requerimientos y que no incluyan las palabras clave no serán listadas. En segundo lugar, Google tampoco rastrea el contenido de archivos tipo vídeo, imágenes o documentos pdf protegidos. Las limitaciones expuestas deberán tenerse en cuenta en la interpretación de los resultados. No obstante, el hecho de que las páginas web de las empresas de turismo activo puedan contener información sobre RSC no visible para los motores de búsqueda, se presentaría como un indicador sobre la falta de comunicación efectiva de la RSC, ya sea de manera consciente o inconsciente por el desconocimiento del funcionamiento de los motores de búsqueda y/o el diseño efectivo de sitios web.

Destacar que la intencionalidad del estudio es analizar la presencia del concepto de la RSC en los sitios web, independientemente de que las empresas de turismo activo tengan una estrategia de RSC o no. Pues lo que se pretende valorar, es la información que se encuentra accesible a los stakeholders, dado que desde un punto de vista pragmático, el resultado de no tener una estrategia de RSC y el de tenerla y no comunicarla de manera efectiva será el mismo. Aun así, cabe decir que las empresas que ofrecen AFMN, por la naturaleza de sus actividades, los espacios en los que tienen lugar dichas actividades, así como los colectivos a los que se dirigen, tienen parte de los valores que se promueven en la RSC en su ADN.

\section{Resultados}

La presencia del término Responsabilidad Social Corporativa, ya sea en su acrónimo (RSC) o en uno de sus términos semejantes también analizados (RSE y Desarrollo Sostenible), se da en el 15\% de las empresas de turismo activo. El término Desarrollo Sostenible es el más utilizado llegando al $13 \%$, lejos del $2 \%$ de empresas que hacen referencia a la RSE y el $2 \%$ a la RSC. La mediana de veces en las que se menciona la RSE por cada sitio web es de 2.0, mientras que en el caso de la RSC es del 1.5 y el Desarrollo Sostenible es de 1.0. (Ver tabla 1).

La ubicación del término es un elemento importante a analizar, pues ésta puede ser un indicador del grado de importancia que una empresa otorga al concepto de la RSC. En este sentido, en el $60 \%$ de los casos los términos se encuentran dentro de la presentación de la empresa o de sus servicios. 
Una empresa menciona el Desarrollo Sostenible en los contenidos a trabajar durante una actividad dirigida a los escolares: "Conocer y apreciar la importancia del agua para el desarrollo sostenible". Otra empresa menciona el Desarrollo Sostenible como elemento que incorpora en sus proyectos: "Encontrar medios prácticos basados en su mayoría en la experiencia directa acumulada a lo largo de todos estos años para revertir los problemas ambientales consiguiendo un desarrollo sostenible a través de planes estratégicos de desarrollo sustentable; planificación territorial; uso de parques naturales y áreas protegidas; (...)"

Tabla 1.

Presencia de la RSC en los sitios webs.

\begin{tabular}{lccc}
\hline & \multicolumn{2}{c}{ Presencia } & $\begin{array}{c}\text { Mediana aparición en } \\
\text { cada sitio web }\end{array}$ \\
\hline Responsabilidad Social Corporativa y RSC & $(\mathbf{n})$ & $(\%)$ & 2.5 \\
Responsabilidad Social Empresarial y RSE & $7 / 310$ & $2.3 \%$ & 1.0 \\
Desarrollo Sostenible & $7 / 310$ & $2.3 \%$ & 1.0 \\
Total sitios web con alguno de los tres términos & $41 / 310$ & $13.2 \%$ & - \\
\hline
\end{tabular}

En relación a la aparición en el mapa de los sitios web, listado de URL's que contiene todas las páginas de un sitio web para su indexación, la presencia es tan solo del $16 \%$. Generalmente los términos aparecen en el mapa web cuando se les dedica una sección o apartado. En este sentido, se cita el contenido que una empresa menciona en su sección de RSC: "(nombre empresa) es una empresa comprometida con la sociedad y el medio ambiente, participando en proyectos que consolidan el tejido asociativo provincial y desarrollando estrategias medioambientales que garanticen la sostenibilidad de todas nuestras acciones".

El término es utilizado como etiqueta (metatag), instrumento para resumir el contenido web e indexarlo, en un $7 \%$ de las ocasiones. Finalmente, en el $51 \%$ de veces los términos aparecen en el contenido de noticias, que en ocasiones no tienen que ver con los servicios que ofrece la empresa, pues se trata de información sobre otras organizaciones. Una empresa menciona la RSC en una de sus noticias sobre un evento que ha organizado: "En este evento de RSC (Responsabilidad Social Corporativa) en concreto, tenemos que agradecer el esfuerzo de todos los asistentes, alrededor de cincuenta empleados y familiares que se sumaron a esta iniciativa, realizando la plantación de más de 70 árboles (algarrobos), cuyo fin era reforestar una zona desbastada por un incendio hace más de 10 años". Otra empresa habla de uno de sus clientes "pretende dar continuidad, dentro de su estrategia de responsabilidad social empresarial, al deporte como instrumento promotor de un estilo de vida saludable, sobre todo en edades tempranas, inculcando a los más jóvenes la importancia de la práctica deportiva y de una alimentación equilibrada".

\section{Discusión y conslusiones}

Basándonos en estos resultados, se pude observar una escasa presencia del concepto de RSC en los contenidos de los sitios web de las empresas de turismo activo de Andalucía, a pesar de que se reclama la incorporación de ésta en la forma de gestionar las actividades deportivas en el medio natural (Funollet et al., 2016; Quintana \& Gregory, 2013) y de que el entorno online es la principal plataforma de comunicación de las empresas de turismo (Cao \& Yang, 2016; Corigliano \& Baggio, 2006). El estudio ha identificado un uso más extendido del término Desarrollo Sostenible por delante de la RSC y la RSE. Esto puede deberse al hecho de que durante mucho tiempo el concepto de Desarrollo Sostenible se ha vinculado principalmente a los retos medio ambientales, poniendo poca atención a los aspectos económicos y sociales (Behringer \& Szegedi, 2016) tal y como se intuye que puede estar sucediendo en las empresas de turismo activo. Esto significaría, que en el caso de existir una intencionalidad hacia la RSC, podría ser que no se estuviera implementando de manera integral 
llegando a otras áreas como la gestión de los recursos humanos, la transparencia, el desarrollo social, etc. De esta limitada interpretación de la RSC, la mayoría de veces centrada en la filantropía, han alertado autores como Lin-Hin (2010). La poca importancia concedida a la RSC también se deduce de la casi inexistencia de una sección dentro del sitio web dedicada a ésta.

Dado que este artículo ha focalizado sobre la presencia de la RSC en los sitios webs de las empresas de turismo activo, sin entrar a valorar si estas disponen de una estrategia de RSC o no, sería interesante realizar una investigación complementaria en esta línea. También, se necesitan investigaciones que entren a analizar en profundidad el contenido de las comunicaciones en materia de RSC y su vinculación con las actividades ofertadas a los grupos escolares. Por último, este estudio podría reproducirse en otros territorios con el objeto de realizar un estudio comparativo.

Los resultados de este estudio proporcionan un soporte al argumento de que existe una necesidad de planificar, implementar y comunicar de manera eficiente una gestión integral de la RSC en las empresas de turismo activo de Andalucía. Los datos presentados han demostrado una escasa presencia del término de la RSC en los sitios webs corporativos, así como una interpretación limitada que focaliza en aspectos medio ambientales sin entrar en la esfera económica y social. Estos hallazgos deberían ser el punto de partida para investigación adicional que contribuya a la generación de conocimiento que posibilite un desarrollo sostenible de la industria de turismo activo. De esta manera, la industria podrá dar respuesta tanto a la imperante necesidad de proteger el medio ambiente, área en la que por el tipo de actividad desarrollada puede darse un impacto más negativo, como a la exigencia de un consumidor cada vez más informado y concienciado que exige responsabilidades a las empresas más allá de las obligaciones legales. La población escolar representa las futuras generaciones de consumidores, y las AFMN tienen un gran potencial para educar en los principios que promueve la RSC, especialmente en el área de medio ambiente. Por ello, las empresas de turismo activo deben dar a conocer su posicionamiento respecto a la RSC, las acciones que llevan a cabo y como integran sus principios en la oferta dirigida a la población escolar. Está información ayudará a los docentes que quieran organizar AFMN a seleccionar a las empresas de turismo activo bajo criterios de responsabilidad social.

\section{Referencias}

Baena, A., \& Granero, A. (2014). Educación física a través de la educación de Aventura. Tándem, 45, 1-7.

Baena, A., Granero, A., \& Ortiz, M. (2012). Quasi-experimental study of the effect of an adventure education programme on classroom satisfaction, physical self-concept and social goals in physical education. Psychologica Belgica, 52(4), 369-386.

Baena, A., Gómez, M. \& Granero, A. (2008). La sostenibilidad del medio ambiente a través de las actividades físico-deportivas en el medio natural y su importancia en la educación ambiental. Investigación Educativa, 12(22), 173-193.

Basch, C. E. H., Zybert, P., Reeves, R., \& Basch, C. E. H. (2017). What do popular YouTube TM videos say about vaccines? Child: Care, Health and Development, 1-5. http://doi.org/10.1111/cch.12442

Behringer, K., \& Szegedi, K. (2016). The role of CSR in achieving sustainable development - theoretical approach. European Scientific Journal, 12(22), 10. http://doi.org/10.19044/esj.2016.v12n22p10

Bowen, H. (1953). Social Responsibilities of the Businessman. New York: Harper \& Row.

Caballero, P., \& Delgado, M.A. (2014). Design of a positive development program through outdoor activities. Journal of Sport and Health Research. 6(1), 29-46.

Cao, K., \& Yang, Z. (2016). A study of e-commerce adoption by tourism websites in China. Journal of Destination Marketing and Management, 5(3), 283-289. http://doi.org/10.1016/j.jdmm.2016.01.005

Carroll, A. B. (1979). A Three-Dimensional Conceptual Model of Corporate Performance. Academy of Management Review, 4(4), 497-505. http://doi.org/10.5465/AMR.1979.4498296

Carroll, A. B. (1991). The Pyramid of Corporate Social Responsibility: Toward the Moral Management of Organizational Stakeholder. Business Horizons, 34(4), 39-48. http://doi.org/10.1016/00076813(91)90005-G 
Comisión Europea. (2001). El libro verde. Libro Verde. Fomentar un marco europeo para la responsabilidad social de las empresas. Brussels. Retrieved from http://scholar.google.com/scholar?hl=en\&btnG=Search\&q=intitle:Green+Book\#8\%5Cnhttp://scholar.g oogle.com/scholar?hl=en\&btnG=Search\&q=intitle:Libro+Verde\#1

Corigliano, M. A., \& Baggio, R. (2006). On the Significance of Tourism Website Evaluations. In M. Hitz, M. Sigala, \& J. Murphy (Eds.), Information and Communication Technologies in Tourism 2006: Proceedings of the International Conference in Lausanne, Switzerland, 2006 (pp. 320-331). Vienna: Springer Vienna. http://doi.org/10.1007/3-211-32710-X_43

Cornelissen, J. (2014). Corporate Communication. A guide to theory and practice (4th ed.). London: Sage.

Elkington, J. (2004). Enter the triple bottom line. In A. Henriques, J. Richardson, \& I. NetLibrary (Eds.), The triple bottom line, does it all add up?: assessing the sustainability of business and CSR (Vol. 1, pp. 116). London: Earthscan. http://doi.org/10.1021/n1034968f

Evans, H., Chao, M. G., Leone, C. M., Finney, M., \& Fraser, A. (2016). Content analysis of web-based norovirus education materials targeting consumers who handle food: An assessment of alignment and readability. Food Control, 65, 32-36. http://doi.org/10.1016/j.foodcont.2016.01.003

Fatma, M., Rahman, Z., \& Khan, I. (2016). Measuring consumer perception of CSR in tourism industry: Scale development and validation. Journal of Hospitality and Tourism Management, 27, 39-48. http://doi.org/10.1016/j.jhtm.2016.03.002

Forética. (2002). Responsabilidad Social Empresarial. Informe Forética 2002. desde la gestión, lo social y ambiental. Bogotá: … Madrid: Forética. Retrieved from http://www.academia.edu/download/43286201/CSR_Corporate_Social_Responsibility.docx

Funollet, F., Inglés, E., \& Labrador, V. (2016). Hacia un nuevo paradigma de la actividad deportiva en el medio natural. Apunts. Educación Física Y Deportes, 124(2), 114-121. http://doi.org/10.5672/apunts.20140983.es.(2016/2).124.13

Galan, M. M., Galera, C., \& Valero, V. (2017). Cause-Related Marketing in Spain. A Consumer Behavior Perspective. Effects on Satisfaction and Loyalty. In C. C.L. (Ed.), The Customer is NOT Always Right? Marketing Orientationsin a Dynamic Business World. Developments in Marketing Science: Proceedings of the Academy of Marketing Science (p. 442). New York: Springer.

Hernández, R., Fernández, C., \& Baptista, M. del P. (2010). Metodología de la investigación (5th ed.). México: McGraw Hill.

Hetze, K., \& Winistörfer, H. (2016). CSR communication on corporate websites compared across continents. International Journal of Bank Marketing, 34(4), 501-528. http://doi.org/10.1108/IJBM-02-2015-0022

Kastenholz, E. (2009). Turismo accesible como responsabiliad social en las empresas y destinos turísticos. El caso de Lousã (Portugal). Rotur: Revista de Ocio Y Turismo, 2, 175-194.

Kwok, T. M. Y., Singla, A. A., Phang, K., \& Lau, A. Y. S. (2017). YouTube as a source of patient information for varicose vein treatment options. Journal of Vascular Surgery: Venous and Lymphatic Disorders, 5(2), 238-243. http://doi.org/10.1016/j.jvsv.2016.10.078

Lin-Hi, N. (2010). The problem with a narrow-minded interpretation of CSR. Why CSR has nothing to do with philantropy. Ramon Llull Journal of Applied Ethics, 1(1), 79-95.

Mediavilla, L., \& Gómez, V. (2016). El turismo activo en España. Identificación de la calidad del servicio en las empresas. Apunts. Educación Física y Deportes, 124(2), 108-113. http://doi.org/10.5672/apunts.20140983.es.(2016/2).124.12

Observatorio de Responsabiliad Social Corporativa. (2014). Introducción a la Responsabilidad Social Corporativa. Madrid: Observatorio de Responsabiliad Social Corporativa. Retrieved from http://observatoriorsc.org/introduccion-la-responsabilidad-social-corporativa/

Perdomo, Y. (2014). Global Report on Adventure Toursim. Madrid.

Quintana, V., \& Gregory, R. (2013). Some perspective sustainability on some scanned rural and active tourism: the case of the region extremeña. ROTUR: Revista de Ocio Y Turismo, 6, 122-139. Retrieved from http://rotur.es/index.php/rotur/article/view/57

Ruiz, J. B., \& Barnett, G. A. (2015). Exploring the presentation of HPV information online: A semantic network analysis of websites. Vaccine, 33(29), 3354-3359. http://doi.org/10.1016/j.vaccine.2015.05.017

Saat, R. M., \& Selamat, M. H. (2014). An Examination of Consumer's Attitude towards Corporate Social Responsibility (CSR) Web Communication Using Media Richness Theory. Procedia - Social and 
Behavioral Sciences, 155(October), 392-397. http://doi.org/10.1016/j.sbspro.2014.10.311

Sones, M., Grantham, S., \& Vieira, E. T. (2009). Communicating CSR via pharmaceutical company web sites: Evaluating message frameworks for external and internal stakeholders. Corporate Communications: An International Journal, 14(2), 144-157. http://doi.org/10.1108/13563280910953834

United Nations. (2014). Guide to Corporate Sustainability. Shaping a sustainable future. New York: United Nations Global Compact. from http://search.ebscohost.com.ezproxy.liv.ac.uk/login.aspx?direct=true \&db=buh\&AN=11177292\&site=e ds-

live \&scope=site $\% 5 \mathrm{Cnhttp}: / /$ search.proquest.com/docview/210954449?accountid=10218\%5Cnhttp://ww w.ub.uni-koeln.de/openurl?url_ver=Z39.88-2004\&rft_val_fmt=info:ofi

World Commission on Environment and Development. (1987). Our Common Future. Oxford: Oxford University Press. 